\title{
Thermal-diffusive ignition and flame initiation by a local energy source
}

\author{
Carlos Vázquez-Espí
}

Amable Liñán

\begin{abstract}
The ignition and flame initiation in a gaseous reacting mixture subject to a local source of thermal energy is analysed by means of large activation energy asymptotics. The ignition transient is assumed to be long enough for heat conduction to be the dominant cooling mechanism. We show the existence of a critical value of the Damköhler number, defined as the ratio of appropriate characteristic times of conduction and chemical reaction, such that ignition only occurs for supercritical values. Additional conditions are required to ensure self-propagation of a flame after ignition. These are obtained, with the thermal-diffusive model, for a source of energy represented by an instantaneous point, line or planar source. The analysis, involving an unsteady free-boundary problem, shows that the initial flame kernel evolves to a self-propagating flame only if the energy released by the source is greater than a critical value.
\end{abstract}

\section{Introduction}

A gaseous reacting mixture can be ignited by a localized deposition of energy that raises the temperature in a small region of the gas, where the times required for the typical reactions occurring in combustion are strongly decreased, since their rates grow greatly with temperature. For ignition to be successful, the rate of chemical heat release must exceed the rate of cooling of the hot spot by either expansion waves or heat conduction.

The first mechanism, associated with fast ignition events, was analysed in a companion paper [16]. In that case the characteristic times of external heating and chemical reaction were short enough to be comparable to the time required by the acoustic waves to travel across the hot spot. For subcritical values of the ratio of the acoustic and reaction times, the cooling effects associated with the expansion waves lower rapidly the temperature in the hot spot. The peak temperature decreases, in a time of the order of the acoustic time, from the peak value $T_{\mathrm{m}}$, resulting from the external heat release, to a lower value $T_{\mathrm{m}}^{\prime}$, which leads to a much larger ignition time. 
In this paper we shall consider the diffusive ignition, in which, after the deposition of energy, the cooling associated with heat conduction across the hot spot, with a characteristic time $t_{\mathrm{c}}$, competes with the chemical heat release, with a characteristic time $t_{\mathrm{ch}}$ determined by the maximum temperature at the hot spot, to define the conditions for ignition. The heat conduction time is much larger than the acoustic time, $t_{\mathrm{a}}$, since the ratio $t_{\mathrm{a}} / t_{\mathrm{c}}$ is also the Knudsen number, the ratio of the mean free path to the size of the hot spot, which is typically very small compared with unity. In this slow, diffusive regime we find ignition at times of order $t_{\mathrm{ch}}$ if the Damköhler number, $t_{\mathrm{c}} / t_{\mathrm{ch}}$, is larger than a critical value; i.e. if the conductive heat losses from the hot spot to the surroundings are overcome by the heat released by the chemical reaction. Although this ignition problem has already been treated by Berman et al [1], it will be revisited in section 2 , with the aim of clarifying the model, completing their results and introducing the flame initiation problem.

After the thermal runaway, which defines the ignition time, the limiting reactant is rapidly depleted in the core of the hot spot; see Dold [5]. However, the achievement of ignition does not guarantee the formation of a self-sustained travelling combustion front, detonation or deflagration, which requires additional conditions. Thus, in the nondiffusive regime, Short [14] has derived the critical conditions leading to a ZND detonation in a reactive mixture subject to small disturbances; He and Clavin [8] and Eckett [6] have calculated the minimum energy to be released by an instantaneous point source in order to develop a detonation wave. When, in the diffusive regime, we consider heated spots with a size large compared with the thickness of the planar flame, we may expect that the conditions for ignition will also ensure flame propagation. The energy required for ignition will increase with the size of the hot spot, so that if we reduce this size, to minimize the ignition energy, to values smaller than the flame thickness, we may not succeed in establishing a flame unless we use energies much larger than those required for ignition by hot spots of small size.

A full detailed analysis of the transition from diffusive ignition to a self-propagating flame, similar to that of Kapila [11], appears to be very involved if we want to account for initial perturbations of arbitrary amplitude, because the process will be strongly dependent on the specific initial conditions. But if we are mainly concerned with the determination of the minimum energy for flame generation, we can consider a simpler approach, close to that of $[6,8]$, assuming that the initial energy is released by an instantaneous point source. In this case the high temperatures at the very beginning after the deposition of energy, represented by the mathematical singularity of the temperature distribution, produce, for Arrhenius reactions, the instantaneous ignition of the mixture and the appearance of a combustion front. Thus, the problem is reduced to the analysis of the dynamics of the front.

For point-source initiation the steady flame-ball solution of Zel'dovich et al [17], although unstable, plays an important role. Without the effects of the heated core, we can expect that flames with a radius larger than the Zel'dovich radius, which depends on the Lewis number, grow and those with a lower radius collapse. The Zel'dovich flame-ball concept can be used to analyse the spherical flame initiation, which will be successful only if the flame kernel, established by the released energy, reaches a radius larger than the critical radius. Joulin [9] (see also $[2-4,10]$ ) assumes that the characteristic time for the evolution of the kernel is determined by the time for spontaneous evolution of a flame around the equilibrium state. For light reactants this time is very large, and the flow field can be divided into two regions: a quasi-steady nearfield, where the main structure of the flame is located, and an unsteady far-field. The matching between both leads to an evolution equation for the flame radius. Numerical experiments with this model allow one to obtain critical conditions for noninstantaneous sources.

Recently, $\mathrm{He}$ [7] has considered a different approach in which the flame-ball does not play a central role, focusing the analysis on the critical conditions for the existence of a self-sustained 
expanding flame. Using a quasi-steady model, He shows that for mixtures with large Lewis numbers such a flame can only exist if its radius is larger than a critical radius, which is smaller than the corresponding flame-ball radius (for small Lewis numbers critical and flameball radius are the same). To obtain a successful initiation the external energy source must sustain the expanding flame kernel until it reaches the critical radius, from which the flame will self-propagate. For the quasi-steady model to be consistent the duration of the energy source must be very long, which limits the scope of the analysis.

The flame initiation problem will be considered in section 3 , in the frame of the thermaldiffusive model, but without restricting ourselves to a quasi-steady description. As a result we shall find the critical energy for successful flame initiation for a symmetrical (planar, line or point) configuration of the source and arbitrary Lewis numbers close to unity.

\section{Diffusive, or slow ignition, regime}

Let us consider a gaseous reacting mixture of two reactants, $\mathrm{F}$ and $\mathrm{O}$, diluted in an inert gas at the initial conditions $\rho_{0}, p_{0}$ and $T_{0}$. This uniform state is perturbed by the addition of an amount of energy, $\dot{Q}(r, t)$ per unit volume and time, in a domain of characteristic length $r_{\mathrm{h}}$. We assume that the energy addition takes place during a finite time interval $t_{\mathrm{e}}$ large compared with the acoustic time $t_{\mathrm{a}}^{\prime}=r_{\mathrm{h}} / \sqrt{\gamma R T_{0} / M}$, where $R / M$ is the gas constant, but short compared with both the homogeneous ignition time evaluated at the maximum temperature resulting from the energy addition and the conduction time $t_{\mathrm{c}}^{\prime}=r_{\mathrm{h}}^{2} / D_{T}$, where $D_{T}$ is the initial value of the thermal diffusivity. Therefore, when describing the ignition process, which involves the timescale $t_{\mathrm{c}}^{\prime}$, the energy deposition appears to take place instantaneously without chemical reaction effects. Since $t_{\mathrm{a}}^{\prime} \ll t_{\mathrm{e}} \ll t_{\mathrm{c}}^{\prime}$, after the external energy deposition the gas conditions are given by

$$
t_{\mathrm{e}} \ll t \ll t_{\mathrm{c}}^{\prime}: \quad T=T_{\mathrm{h}}(r), \quad p=p_{0}, \quad \rho=\rho_{\mathrm{h}}(r),
$$

where $\rho_{\mathrm{h}}=p_{0} M / R T_{\mathrm{h}}$. The temperature distribution, $T_{\mathrm{h}}(r)$, after the energy addition can be numerically calculated as a function of $\dot{Q}(r, t)$. In our analysis of the ignition stage we shall consider $T_{\mathrm{h}}(r)$ as a given symmetrical, bell-shaped function of $r$, with the scale $r_{\mathrm{h}}$ such that $T_{\mathrm{h}} / T_{0}-1 \rightarrow 0$ as $r / r_{\mathrm{h}} \rightarrow \infty$. $T_{\mathrm{h}}$ has its maximum value $T_{\mathrm{m}}=T_{\mathrm{h}}(0)$ at $r=0$.

The chemical reaction between fuel and oxidizer is modelled by a single reaction $\mathrm{F}+s \mathrm{O} \rightarrow P+q$, where $s$ and $q$ stand for the mass of oxidizer consumed and heat released, respectively, per unit mass of fuel. The fuel consumption rate is given by an Arrhenius law, with activation energy $E$ much larger than the thermal energy $R T$, written in the form

$$
w=\rho Y_{\mathrm{F}}^{n_{\mathrm{F}}} Y_{\mathrm{O}}^{n_{\mathrm{O}}} B \mathrm{e}^{-E / R T},
$$

where $Y_{\mathrm{F}}$ and $Y_{\mathrm{O}}$ are the mass fractions of fuel and oxidizer, $n_{\mathrm{F}}$ and $n_{\mathrm{O}}$ are the reaction orders, and $B$ is the pre-exponential factor.

Therefore, the equations describing the ignition and symmetrical flame propagation process take the following form:

$$
\begin{aligned}
& p_{0}=\rho T R / M, \\
& \frac{\partial \rho}{\partial t}=-\frac{1}{r^{j}} \frac{\partial}{\partial r}\left(r^{j} \rho u\right), \\
& \rho c_{p} \frac{D T}{D t}=\frac{1}{r^{j}} \frac{\partial}{\partial r}\left(r^{j} k \frac{\partial T}{\partial r}\right)+q w
\end{aligned}
$$




$$
\begin{aligned}
& \rho \frac{D Y_{\mathrm{F}}}{D t}=\frac{1}{r^{j}} \frac{\partial}{\partial r}\left(r^{j} \rho D_{\mathrm{F}} \frac{\partial Y_{\mathrm{F}}}{\partial r}\right)-w, \\
& \rho \frac{D Y_{\mathrm{O}}}{D t}=\frac{1}{r^{j}} \frac{\partial}{\partial r}\left(r^{j} \rho D_{\mathrm{O}} \frac{\partial Y_{\mathrm{O}}}{\partial r}\right)-s w,
\end{aligned}
$$

where $D / D t=\partial / \partial t+u \partial / \partial r$ is the material derivative. In the symmetrical form of these equations we have not included the momentum equation. This is used to estimate the order, $\rho_{0}\left(r_{\mathrm{h}} / t_{\mathrm{c}}^{\prime}\right)^{2}$, of the spatial pressure variations, which are very small compared with $p_{0}$, allowing us to simplify the equation of state to the form (3). We shall consider the mean molecular mass $M$ to be constant. We have used Fick's law to calculate the diffusion fluxes, with mass diffusion coefficients $D_{\mathrm{F}}$ and $D_{\mathrm{O}}$ of fuel and oxidizer, respectively. We also assume that $D_{\mathrm{F}}$ and $D_{\mathrm{O}}$, the specific heat $c_{p}$ and the thermal conductivity $k$ to be constant. These equations must be supplemented with the following initial and boundary conditions:

$$
\begin{array}{ll}
t=0: & T-T_{\mathrm{h}}=Y_{\mathrm{F}}-Y_{\mathrm{F}, 0}=Y_{\mathrm{O}}-Y_{\mathrm{O}, 0}=0, \\
r=0: & u=\frac{\partial T}{\partial r}=\frac{\partial Y_{\mathrm{F}}}{\partial r}=\frac{\partial Y_{\mathrm{O}}}{\partial r}=0, \\
r \rightarrow \infty: & T-T_{0}=Y_{\mathrm{F}}-Y_{\mathrm{F}, 0}=Y_{\mathrm{O}}-Y_{\mathrm{O}, 0}=0 .
\end{array}
$$

In absence of diffusion and heat conduction effects, the chemical reaction would produce a thermal runaway at a time $t_{\mathrm{R}}$ defined by $t_{\mathrm{R}}(r)=\left(c_{p} R T_{\mathrm{h}}^{2}(r) / q E B Y_{\mathrm{F}, 0}^{n_{\mathrm{F}}} Y_{\mathrm{O}, 0}^{n_{\mathrm{O}}}\right) \mathrm{e}^{E / R T_{\mathrm{h}}(r)}$. Its minimum value is $t_{\mathrm{ch}}=t_{\mathrm{R}}(0)$, given by

$$
t_{\mathrm{ch}}=\frac{R T_{\mathrm{m}}}{E} \frac{c_{p} T_{\mathrm{m}}}{q Y_{\mathrm{F}, 0}^{n_{\mathrm{F}}} Y_{\mathrm{O}, 0}^{n_{\mathrm{O}}}} B^{-1} \mathrm{e}^{E / R T_{\mathrm{m}}},
$$

much shorter than the value $t_{\infty}=t_{\mathrm{R}}(\infty)$, associated with the initial temperature $T_{0}$. Notice that the conditions far from the hot spot are time dependent due to the reaction which takes place homogeneously and could result in a self-ignition time $t_{\infty} / \gamma=\left(c_{v} R T_{0}^{2} / q E B Y_{\mathrm{F}, 0}^{n_{\mathrm{F}}} Y_{\mathrm{O}, 0}^{n_{\mathrm{O}}}\right) \mathrm{e}^{E / R T_{0}}$. However, $t_{\infty}$ is exponentially large compared with $t_{\mathrm{ch}}$ if the Zel'dovich number holds $E\left(T_{\mathrm{m}}-T_{0}\right) / R T_{\mathrm{m}}^{2} \gg 1$, which allows us to consider (10) as the appropriate boundary conditions at $r \rightarrow \infty$ during the ignition period.

The homogeneous chemical time, $t_{\mathrm{R}}$, is of order $t_{\mathrm{ch}}$ only in the Frank-Kamenetskii region, located at the centre of the hot spot, where $T_{\mathrm{h}}(r)-T_{\mathrm{m}} \sim R T_{\mathrm{m}}^{2} / E \ll T_{\mathrm{m}}$ if $\varepsilon=R T_{\mathrm{m}} / E \ll 1$. In this region the chemical reaction will produce changes in temperature of order $R T_{\mathrm{m}}^{2} / E$ at times of order $t_{\mathrm{ch}}$. Outside the Frank-Kamenetskii region the temperature is lower and the reaction appears to be frozen at times $t \sim t_{\mathrm{ch}}$.

If we admit that the hot spot produced by the external energy release is not flat at the centre, we can define the characteristic size $r_{\mathrm{h}}$ in terms of the curvature of the initial profile as $r_{\mathrm{h}}^{2}=-2 T_{\mathrm{m}} /\left(\mathrm{d}^{2} T_{\mathrm{h}} / \mathrm{d} r^{2}\right)_{r=0}$. The characteristic size of the Frank-Kamenetskii region, $r_{\mathrm{c}}$, is then given by $r_{\mathrm{c}}=\sqrt{\varepsilon} r_{\mathrm{h}}$, so that the initial temperature in the core region of the hot spot is given by $T_{\mathrm{h}}=T_{\mathrm{m}}\left(1-\varepsilon\left(r / r_{\mathrm{c}}\right)^{2}\right)$. For small times compared with $t_{\mathrm{c}}^{\prime}$ and $r \sim r_{\mathrm{c}}$ the inert temperature, given by the solution of the problem (3)-(10) with $B=0$, reads

$$
\frac{T_{\mathrm{I}}-T_{\mathrm{m}}}{\varepsilon T_{\mathrm{m}}}=-\left(\frac{r}{r_{\mathrm{c}}}\right)^{2}-2(1+j)\left(\frac{t}{t_{\mathrm{c}}}\right),
$$

where $t_{\mathrm{c}}=r_{\mathrm{c}}^{2} / D_{T}$ is the conduction time through the Frank-Kamenetskii region, required to decrease the inert temperature by an amount of order $\varepsilon T_{\mathrm{m}}$. If $t_{\mathrm{ch}} \gg t_{\mathrm{c}}$, the heat released by the source is conducted outside the core of the hot spot, and the chemical reaction will be frozen without significant thermal effects. Hence, ignition will not occur at times of order $t_{\mathrm{c}}$. 
Conversely, if $t_{\mathrm{ch}} \ll t_{\mathrm{c}}$, ignition occurs at a time $t=t_{\mathrm{ch}}$ before conductive losses of energy appear. Finally, if $t_{\mathrm{ch}} \sim t_{\mathrm{c}}$ there is a competition between heat conduction and chemical reaction and we will find an ignition event for values of the Damköhler number

$$
\delta=\frac{t_{\mathrm{c}}}{t_{\mathrm{ch}}}=\frac{r_{\mathrm{h}}^{2}}{D_{T}} \frac{q Y_{\mathrm{F}, 0}^{n_{\mathrm{F}}} Y_{\mathrm{O}, 0}^{n_{\mathrm{O}}}}{c_{p} T_{\mathrm{m}}} B \mathrm{e}^{-E / R T_{\mathrm{m}}},
$$

larger than a critical value of order unity to be determined.

To describe the evolution of the reacting mixture in the Frank-Kamenetskii region, $r \sim r_{\mathrm{c}}$, for times $t \sim t_{\mathrm{c}} \sim t_{\mathrm{ch}}$ we use the variables

$\xi=\frac{r}{r_{\mathrm{c}}}, \quad \tau=\frac{t}{t_{\mathrm{c}}}, \quad v=\frac{u-u_{\mathrm{I}}}{\left(\varepsilon r_{\mathrm{c}} / t_{\mathrm{c}}\right)}, \quad \hat{\rho}=\frac{\rho-\rho_{\mathrm{I}}}{\varepsilon \rho_{\mathrm{m}}}, \quad \varphi=\frac{T-T_{\mathrm{I}}}{\varepsilon T_{\mathrm{m}}}$,

where the inert solutions $\rho_{\mathrm{I}}$ and $u_{\mathrm{I}}$, readily obtained from (3)-(10), are given by $\rho_{\mathrm{I}}=$ $\rho_{\mathrm{m}}\left(1+\varepsilon\left(\xi^{2}+2(1+j) \tau^{2}\right)\right)$ and $u_{\mathrm{I}}=-2 \varepsilon\left(r_{\mathrm{c}} / t_{\mathrm{c}}\right) \xi$. When equations (3)-(7) are written in the new variables we observe that all convective terms are of order $\varepsilon$, so that they can be neglected in first approximation during the ignition stage. In addition, the equations for the mass fractions show that their changes are also of order $\varepsilon$, so that in first approximation we can write $Y_{\mathrm{F}}=Y_{\mathrm{F}, 0}$ and $Y_{\mathrm{O}}=Y_{\mathrm{O}, 0}$. Therefore, the nondimensional increment in temperature $\varphi$, due to the chemical reaction, is given by the nonlinear problem

$$
\begin{aligned}
& \frac{\partial \varphi}{\partial \tau}=\frac{1}{\xi} \frac{\partial}{\partial \xi}\left(\xi^{j} \frac{\partial \varphi}{\partial \xi}\right)+\delta \exp \left(\varphi-\xi^{2}-2(1+j) \tau\right), \\
& \varphi(0, \xi)=\varphi_{\xi}(\tau, 0)=\varphi(\tau, \infty)=0 .
\end{aligned}
$$

Once $\varphi$ has been computed, the density and velocity disturbances are given by the equation of state, $\varphi+\hat{\rho}=0$, and the continuity equation, $\partial\left(\xi^{j} v\right) / \partial \xi=-\xi^{j} \partial \hat{\rho} / \partial \tau$.

This model of the diffusive ignition, first formulated by Berman et al [1], differs from that described by Kassoy et al [12], who consider small initial temperature disturbances and include the heating by homogeneous compression.

The solution of (15) and (16), giving the temperature rise $\varphi$ due to the chemical reaction, shows blow-up at $\tau=\tau_{\mathrm{i}}(\delta)$ when $\delta$ is larger than the critical value $\delta_{\mathrm{c}}$. For $\delta<\delta_{\mathrm{c}}$ there is no ignition and $\varphi$ evolves to the steady solution $\varphi=0$ corresponding to the frozen solution. To obtain the numerical solution, for a given value of $\delta$, we use the Newton-Kantorovich method. The resulting linear problem was discretized via an implicit finite-volume method, with a nonuniform mesh. The Crank-Nicholson method was used, allowing a variable time-step size to achieve fast convergence. In the supercritical cases the accurate value of the ignition time was obtained from the behaviour $\varphi \sim-\ln \left(\delta \mathrm{e}^{-2(1+j) \tau_{\mathrm{i}}}\left(\tau_{\mathrm{i}}-\tau\right)\right)$ for $\tau_{\mathrm{i}}-\tau \ll 1$ [5]. Figure 1 shows the maximum temperature $\varphi(0, \tau)$ as a function of $\tau$ for different values of $\delta$. The critical values, $\delta_{c}$, of the Damköhler number are 3.2788, 7.6260 and 12.427 for the planar, cylindrical and spherical cases, respectively. Figure 2 shows the ignition time as a function of $\delta$.

The ignition process has a local character and is determined by local properties of the initial temperature profile, i.e. the maximum temperature, $T_{\mathrm{m}}$, and the curvature at the centre of the hot spot where $T=T_{\mathrm{m}}$. Although the kinetics for ignition may involve activation energies and pre-exponential factors different from those appropriate for flame propagation, we shall write here the Damköhler number for the ignition problem in terms of the characteristic parameters of the planar flame: the adiabatic temperature $T_{\mathrm{b}}=T_{0}+q Y_{\mathrm{F}, 0} / c_{p}$ (here we assume that the fuel is the limiting reactant); the Zel'dovich number $\beta=E\left(T_{\mathrm{b}}-T_{0}\right) / R T_{\mathrm{b}}^{2} \gg 1$; and the planar flame velocity $U_{\mathrm{p}}$ or, equivalently, the thickness of the preheat zone $l_{\mathrm{p}}=D_{T} / U_{\mathrm{p}}=$ $\left(\Lambda D_{T} \beta^{n_{\mathrm{F}}+1} \mathrm{e}^{E / R T_{\mathrm{b}}} / 2 B Y_{\mathrm{F}, 0}^{n_{\mathrm{F}}-1} Y_{\mathrm{O}, 0}^{n_{\mathrm{o}}}\right)^{1 / 2}$, where $D_{T}=k / \rho c_{p}$ is the thermal diffusivity and $\Lambda$ is a 


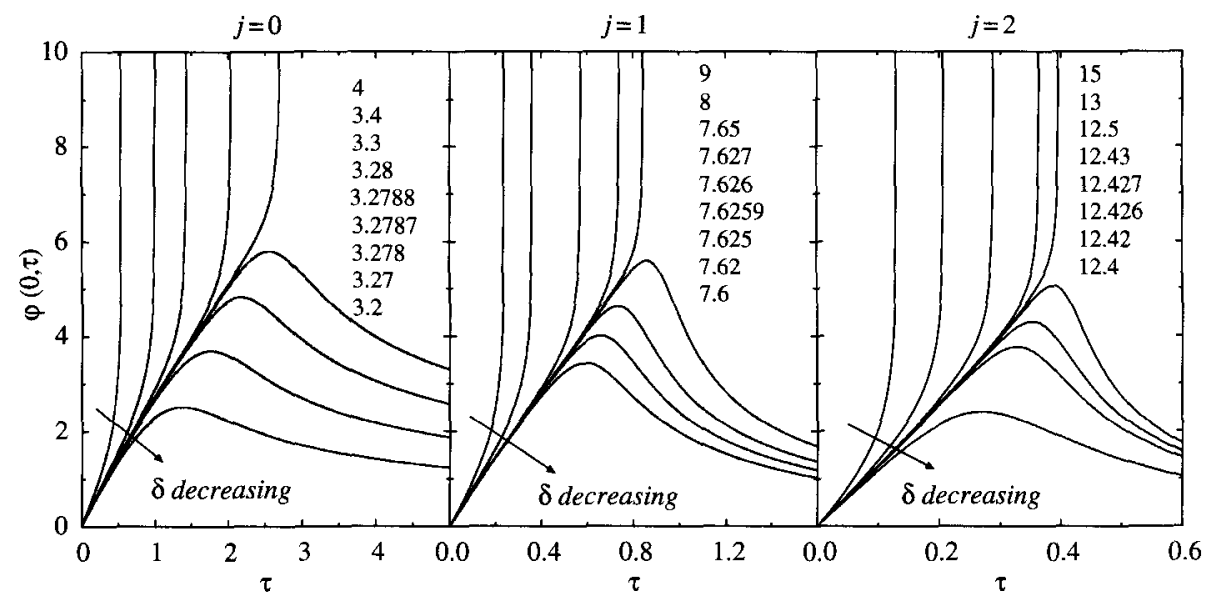

Figure 1. The maximum temperature $\varphi(0, \tau)$ as a function of $\tau$ for several values of $\delta$ (showed in the inset).

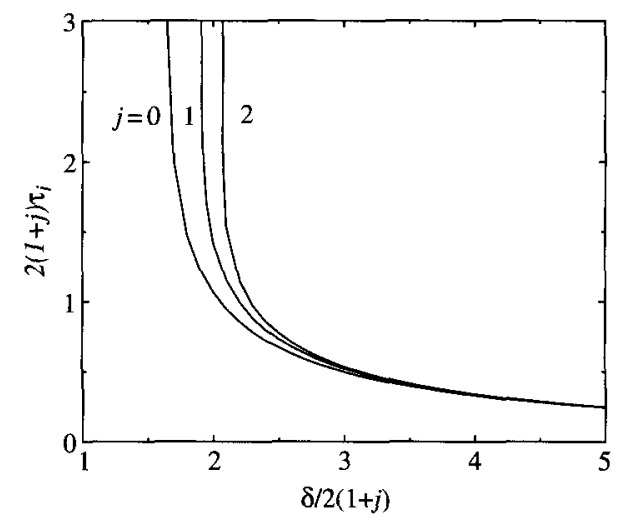

Figure 2. The ignition time $\tau_{\mathrm{i}}$ as a function of $\delta$.

parameter of order unity to be defined later, after equation (26). The Damköhler number $\delta$ can be written in terms of $l_{\mathrm{p}}$ and the hot-spot size $r_{\mathrm{h}}$, then the condition for ignition is given by

$$
\frac{\Lambda}{2}\left(\frac{r_{\mathrm{h}}}{l_{\mathrm{p}}}\right)^{2} \beta^{n_{\mathrm{F}}+1} \frac{T_{\mathrm{b}}-T_{0}}{T_{\mathrm{m}}} \exp \left(\beta \frac{T_{\mathrm{b}}\left(T_{\mathrm{m}}-T_{\mathrm{b}}\right)}{T_{\mathrm{m}}\left(T_{\mathrm{b}}-T_{0}\right)}\right)>\delta_{\mathrm{c}} .
$$

For large values of $\beta$ and values of $r_{\mathrm{h}} / l_{\mathrm{p}}$ not very large or very small compared with unity, we obtain ignition if $\left(T_{\mathrm{m}}-T_{\mathrm{b}}\right)>0$, or no ignition if $\left(T_{\mathrm{m}}-T_{\mathrm{b}}\right)<0$; this criterion is practically independent of the ratio $r_{\mathrm{h}} / l_{\mathrm{p}}$, except for unreasonably small or large values of $r_{\mathrm{h}} / l_{\mathrm{p}}$. For given values of $\delta_{\mathrm{c}}, n_{\mathrm{F}}, \alpha=\left(T_{\mathrm{b}}-T_{0}\right) / T_{\mathrm{b}}$ and $\beta$, the relation (17) determines the minimum value of $r_{\mathrm{h}} / l_{\mathrm{p}}$ that leads to ignition for each value of $\left(T_{\mathrm{m}}-T_{0}\right) /\left(T_{\mathrm{b}}-T_{0}\right)$. In figure 3 we have plotted, for $n_{\mathrm{F}}=1, \alpha=0.8$ and $\beta=15,\left(\Lambda / \delta_{\mathrm{c}}\right)^{1 / 2}\left(r_{\mathrm{h}} / l_{\mathrm{p}}\right)_{\min }$ as a function of $\left(T_{\mathrm{m}}-T_{0}\right) /\left(T_{\mathrm{b}}-T_{0}\right)$, showing the abrupt change in $\left(r_{\mathrm{h}} / l_{\mathrm{p}}\right)_{\min }$ around $T_{\mathrm{m}}=T_{\mathrm{b}}$. If the energy released by the external source is $\tilde{E}_{0}$ (per unit surface for $j=0$ and per unit length for $j=1$ ) and the resulting temperature profile is of the Gaussian type, we can write $\tilde{E}_{0} / \rho c_{p}\left(T_{\mathrm{b}}-T_{0}\right) l_{\mathrm{p}}^{1+j} \sim\left(r_{\mathrm{h}} / l_{\mathrm{p}}\right)^{1+j}\left(T_{\mathrm{m}}-T_{0}\right) /\left(T_{\mathrm{b}}-T_{0}\right)$. Hence, although it is possible to achieve ignition with a very small value of the ratio $r_{\mathrm{h}} / l_{\mathrm{p}}$ and $\left(T_{\mathrm{m}}-T_{0}\right) /\left(T_{\mathrm{b}}-T_{0}\right) \sim 1$, the energy 


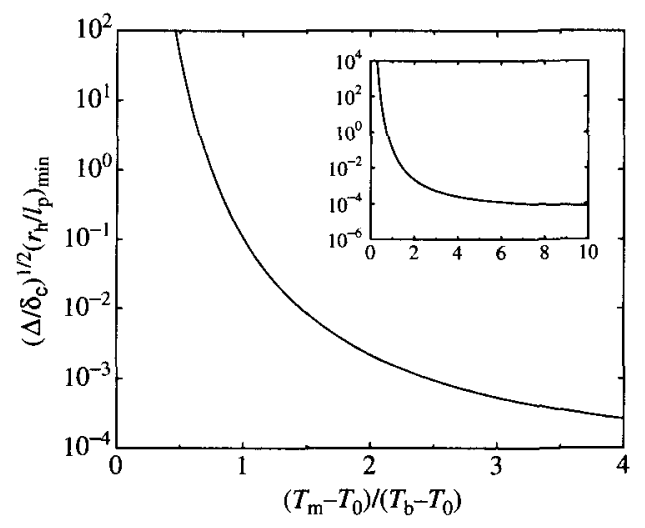

Figure 3. The minimum radius of the hot spot leading to ignition, $\left(\Lambda / \delta_{\mathrm{c}}\right)^{1 / 2}\left(r_{\mathrm{h}} / l_{\mathrm{p}}\right)_{\min }$, as a function of $\left(T_{\mathrm{m}}-T_{0}\right) /\left(T_{\mathrm{b}}-T_{0}\right)$ for $\alpha=0.8$ and $\beta=15$.

content of the hot spot would be so small that might not be enough to develop a self-propagating flame. Notice that all profiles with the same local behaviour at $r=0$ are equivalent from the ignition point of view, but we cannot expect all of them lead to a successful flame initiation.

\section{Minimum energy for flame propagation}

The above analysis has shown the existence, for Arrhenius reactions, of a critical value $\delta_{\mathrm{c}}$ of the Damköhler number, such that only if $\delta>\delta_{\mathrm{c}}$ will ignition occur at times much shorter than the homogeneous ignition time $t_{\infty}$. This is the first condition to be fulfilled in the development of a self-propagating flame. Although necessary, it is not sufficient to ensure a successful flame initiation. Moreover, to describe the flame propagation we must account for the effects of the diffusion and consumption of the reactants, which have been neglected during the ignition stage.

Kapila [11] has described the transition from the ignition regime to the self-propagating regime when a uniform energy flux is applied continuously to the surface of a reactive solid. When the ignition results from the instantaneous deposition of a finite amount of energy, a similar evolution is found after the first stage of thermal runaway. After burning completely the fuel in the centre of the hot spot, we encounter an expanding reactive core, where the fuel has been completely consumed. This core is separated by a thin reaction layer from the outer region where the reaction can be considered frozen. The flame kernel expands at a very high speed, much larger than the planar flame speed corresponding to the initial conditions of the mixture, because of the excess enthalpy associated with the initial external energy addition. With increasing values of the flame radius its velocity decreases because of the lowering values of the excess enthalpy. If the initiating energy is lower than a critical value, the outer motion of the flame front stops and starts to recede to the region of burnt gases. With this contraction stage it is possible to maintain the balance between the supply and consumption of the reactants, because the receding movement of the front decreases the reactant diffusion flux allowing the front to adapt itself to the lowering reaction rate. If the flame continues to move back and, eventually, collapses to the centre of the hot spot, the initiation of the flame fails. Only when the hot spot contains enough energy the flame kernel evolves to an expanding flame that eventually will attain the uniform propagation regime.

According to the analysis by Kapila [11], the events subsequent to the ignition that lead to the development of a flame occur in a time very short compared with the ignition time. Thus, the elapsed time since the deposition of energy until the appearance of a flame kernel 
is approximately given by the ignition time, $t_{\mathrm{i}}$, which is of the same order that the chemical time, $t_{\mathrm{ch}}$, defined by (11). We can use the residence time in the planar flame, $t_{\mathrm{p}}=l_{\mathrm{p}}^{2} / D_{T}$, as the characteristic time to describe the flame propagation. The ratio of both times satisfies

$$
\frac{t_{\mathrm{ch}}}{t_{\mathrm{p}}}=\frac{1}{\beta \delta}\left(\frac{r_{\mathrm{h}}}{l_{\mathrm{p}}}\right)^{2} \frac{T_{\mathrm{m}}\left(T_{\mathrm{b}}-T_{0}\right)}{T_{\mathrm{b}}^{2}},
$$

so that if the condition (17) for ignition holds and $T_{\mathrm{m}}<T_{\mathrm{b}}$, when for ignition to occur the hot-spot radius must be large compared with the flame thickness, i.e. $r_{\mathrm{h}} / l_{\mathrm{p}} \gg 1$, the ignition time is large compared with the residence time in the planar flame; while the opposite is true when $T_{\mathrm{m}}>T_{\mathrm{b}}$, or $r_{\mathrm{h}} / l_{\mathrm{p}} \ll 1$. Thus, in the first case, $T_{\mathrm{m}}<T_{\mathrm{b}}$, the conditions for successful flame initiation are given by the conditions of ignition, which require an energy input $\tilde{E}_{0}$, of order $\rho c_{p}\left(T_{\mathrm{m}}-T_{0}\right) r_{\mathrm{h}}^{j+1}$, that grows rapidly with $T_{\mathrm{b}}-T_{\mathrm{m}}$.

When $T_{\mathrm{m}}$ grows above $T_{\mathrm{b}}$ the energy required for ignition becomes, in agreement with (17), very small and the ignition time becomes very small compared with $t_{\mathrm{p}}$. This time characterizes in these cases the main stage of flame evolution, which defines whether the flame, created just after the ignition thermal runaway, will successfully evolve to an outgrowing flame or begin to collapse and quench. When $T_{\mathrm{m}}>T_{\mathrm{b}}$, or $r_{\mathrm{h}} \ll l_{\mathrm{p}}$, the energy required for flame initiation may be expected to be very large compared with that needed for ignition.

We shall calculate the minimum energy, $\tilde{E}_{0, \mathrm{c}}$, for successful flame initiation in the cases $r_{\mathrm{h}} / l_{\mathrm{p}} \ll 1$, when the flame initiation process depends mainly on what happens to the flame when its radius has grown to values of order $l_{\mathrm{p}}$ at times of order $t_{\mathrm{p}}$. At these times the external energy $\tilde{E}_{0}$ appears to be added instantaneously, as a point source; giving birth to a flame front whose dynamics we must describe to elucidate if, eventually, a self-propagating flame is attained or not. As result of this analysis we expect to find a minimum value $\tilde{E}_{0, \mathrm{c}}$, such that the relation $\tilde{E}_{0}>\tilde{E}_{0, \mathrm{c}}$ can be used as criterion to ensure flame propagation at least when $r_{\mathrm{h}} / l_{\mathrm{p}}$ is small and the instantaneous point source mimics adequately the hot spot.

\subsection{Formulation and dimensionless equations}

With the aim of setting up the problem of describing the evolution of the flame front in its simplest form, we will adopt the thermal-diffusive, or constant density, model in which expansion and convective effects are neglected. Since large increments in temperature are involved, it is obvious that these effects must play a role; consequently, the conclusions of the analysis are expected to be only of qualitative significance. However, in spite of the simplifications this model leads to a nontrivial mathematical free-boundary problem, as we will show in the following. The thermal-diffusive model constitutes a previous and necessary step for the analysis of a convective-diffusive model. The governing equations (5)-(7) simplify, with $u=0$ and $\rho=$ constant, to the system:

$$
\begin{aligned}
& \frac{\partial T}{\partial t}=\frac{1}{r^{j}} \frac{\partial}{\partial r}\left(D_{T} r^{j} \frac{\partial T}{\partial r}\right)+q \frac{w}{\rho c_{p}}, \\
& \frac{\partial Y_{\mathrm{F}}}{\partial t}=\frac{1}{r^{j}} \frac{\partial}{\partial r}\left(D_{\mathrm{F}} r^{j} \frac{\partial Y_{\mathrm{F}}}{\partial r}\right)-\frac{w}{\rho}, \\
& \frac{\partial Y_{\mathrm{O}}}{\partial t}=\frac{1}{r^{j}} \frac{\partial}{\partial r}\left(D_{\mathrm{O}} r^{j} \frac{\partial Y_{\mathrm{O}}}{\partial r}\right)-s \frac{w}{\rho},
\end{aligned}
$$

with $w$ given by (2). At $t=0$ an instantaneous point source (more precisely, a symmetrical $\left(2-j\right.$ )-dimensional distribution) located at $r=0$ releases a finite quantity of heat $\tilde{E}_{0}$. This source can be represented by $\dot{Q}(r, t)=\tilde{E}_{0} \delta(t) \delta(r) / c_{j} r^{j}$ where the factors $c_{j}$ are given by 
$c_{0}=2, c_{1}=2 \pi$ and $c_{2}=4 \pi$ and $\delta(\cdot)$ stands for the Dirac delta function. Without chemical effects, the inert temperature distribution, $T_{\mathrm{I}}$, is given by the solution of the problem

$$
\begin{array}{rlrl}
\rho c_{p} \frac{\partial T_{\mathrm{I}}}{\partial t} & =\frac{1}{r^{j}} \frac{\partial}{\partial r}\left(k r^{j} \frac{\partial T_{\mathrm{I}}}{\partial r}\right), & \frac{\partial T_{\mathrm{I}}(0, t)}{\partial r} & =0, \\
T_{\mathrm{I}}(\infty, t) & =T_{0} ; \quad \int_{0}^{\infty}\left(T_{\mathrm{I}}-T_{0}\right) c_{j} r^{j} \mathrm{~d} r=\tilde{E}_{0} / \rho c_{p} .
\end{array}
$$

We readily find

$$
T_{\mathrm{I}}(r, t)-T_{0}=\frac{\tilde{E}_{0} / \rho c_{p}}{\left(4 \pi D_{T} t\right)^{(1+j) / 2}} \exp \left(-\frac{r^{2}}{4 D_{T} t}\right) .
$$

As result of this form of energy source the temperature distribution at $t=0^{+}$is also a Dirac delta function of $r$, whose evolution for very small times is assumed to model the initial flame kernel: a very small core, where the temperature is high and the chemical reaction is completed, surrounded by cold gas at the initial composition. Therefore we can write the initial conditions for (19)-(21) as

$$
r>0, t=0^{+}: \quad T=T_{I}(r, t), \quad Y_{\mathrm{F}}=Y_{\mathrm{F}, 0}, \quad Y_{\mathrm{O}}=Y_{\mathrm{O}, 0},
$$

which completes the formulation of the problem.

In order to nondimensionalize the problem it is convenient to introduce the following parameters: Lewis numbers $L_{i}=D_{T} / D_{i}(i=\mathrm{F}, \mathrm{O})$; equivalence ratio $\phi=s Y_{\mathrm{F}, 0} / Y_{\mathrm{O}, 0}$ (considering the mixture to be lean $\phi<1$, not close to stoichiometry; the case $\phi>1$ is easily analysed by interchanging the roles of $\mathrm{F}$ and $\mathrm{O}$; the quasi-stoichiometric case, $\phi \simeq 1$, is considered in the appendix); adiabatic temperature $T_{\mathrm{b}}=T_{0}+q Y_{\mathrm{F}, 0} / c_{p}$; heat release parameter $\alpha=q Y_{\mathrm{F}, 0} / c_{p} T_{\mathrm{b}}$; and Zel'dovich number $\beta=E\left(T_{\mathrm{b}}-T_{0}\right) / R T_{\mathrm{b}}^{2} \gg 1$. We will use the following variables:

$\xi=\frac{r}{l_{\mathrm{p}}}, \quad \tau=\frac{t}{t_{\mathrm{p}}}, \quad \varphi=\frac{T-T_{\mathrm{b}}}{T_{\mathrm{b}}-T_{0}}, \quad Y=\frac{Y_{\mathrm{F}}}{Y_{\mathrm{F}, 0}}, \quad X=\frac{Y_{\mathrm{O}}}{\phi Y_{\mathrm{O}, 0}}$,

where $l_{\mathrm{p}}=D_{T} / U_{\mathrm{p}}$ and $t_{\mathrm{p}}=D_{T} / U_{\mathrm{p}}^{2}$ given in terms of the planar flame speed defined by

$$
U_{\mathrm{p}}=\left(2 D_{T} \Lambda^{-1} Y_{\mathrm{F}, 0}^{n_{\mathrm{F}}-1} Y_{\mathrm{O}, 0}^{n_{\mathrm{O}}} \beta^{-\left(n_{\mathrm{F}}+1\right)} B \mathrm{e}^{-E / R T_{\mathrm{b}}}\right)^{1 / 2},
$$

with $\Lambda^{-1}=(1-\phi)^{n_{0}} \Gamma\left(n_{\mathrm{F}}+1\right)$.

The dimensionless inert temperature is given by $\varphi_{\mathrm{I}}(\xi, \tau)=\beta^{-1} E_{0} \theta(\xi, \tau)-1$, where

$\theta(\xi, \tau)=\frac{\mathrm{e}^{-\xi^{2} / 4 \tau}}{\tau^{(1+j) / 2}} \quad$ and $\quad E_{0}=\frac{\tilde{E}_{0}}{\beta^{-1} \rho c_{p}\left(T_{\mathrm{b}}-T_{0}\right)\left(4 \pi l_{\mathrm{p}}^{2}\right)^{(1+j) / 2}}$.

$E_{0}$ is proportional to the ratio between the released energy and $\beta^{-1}$ times the chemical energy contained in a $(1+j)$-dimensional sphere whose radius is $l_{\mathrm{p}}$. The factor $\beta^{-1}$ has been included because, as we will see below (cf equation (32)), an excess enthalpy of order $\beta^{-1}$ changes the reaction rate by a factor of order unity. Therefore, we can expect $E_{0} \sim 1$ to be sufficient for flame initiation.

In the new variables, the conservation equations and initial and boundary conditions take the form

$$
\begin{aligned}
& \frac{\partial \varphi}{\partial \tau}-\Delta \varphi=-\frac{\partial Y}{\partial \tau}+\frac{1}{L_{\mathrm{F}}} \Delta Y=-\frac{\partial X}{\partial \tau}+\frac{1}{L_{\mathrm{O}}} \Delta X=\Omega, \\
& \xi=0: \quad \frac{\partial \varphi}{\partial \xi}=\frac{\partial Y}{\partial \xi}=\frac{\partial X}{\partial \xi}=0, \\
& \xi=\infty: \quad \varphi=-1, \quad Y=1, \quad X=\phi^{-1}, \\
& \xi>0, \tau=0^{+}: \quad \varphi=\beta^{-1} E_{0} \theta(\xi, \tau)-1, \quad Y=1, \quad X=\phi^{-1},
\end{aligned}
$$


where $\Delta$ stands for the symmetric form, $\left(1 / \xi^{j}\right) \partial\left(\xi^{j} \partial / \partial \xi\right) / \partial \xi$, of the Laplacian operator and the dimensionless reaction rate is given by

$$
\Omega=\frac{1}{2} \Lambda \phi^{n_{0}} \beta^{n_{\mathrm{F}}+1} Y^{n_{\mathrm{F}}} X^{n_{0}} \exp \left(\beta \frac{\varphi}{1+\alpha \varphi}\right) .
$$

From equations (28) two conservation equations can be derived for the Shvab-Zel'dovich variables $H \equiv \varphi+Y / L_{\mathrm{F}}$ and $Z \equiv Y / L_{\mathrm{F}}-X / L_{\mathrm{O}}$ which satisfy the following equations, and boundary and initial conditions

$$
\begin{aligned}
& \frac{\partial H}{\partial \tau}-\Delta H=\left(L_{\mathrm{F}}^{-1}-1\right) \frac{\partial Y}{\partial \tau}, \\
& \frac{\partial Z}{\partial \tau}-\Delta Z=\left(L_{\mathrm{F}}^{-1}-1\right) \frac{\partial Y}{\partial \tau}-\left(L_{\mathrm{O}}^{-1}-1\right) \frac{\partial X}{\partial \tau}, \\
& \xi=0: \quad \frac{\partial H}{\partial \xi}=\frac{\partial Z}{\partial \xi}=0, \\
& \xi=\infty: \quad H=L_{\mathrm{F}}^{-1}-1, \quad Z=L_{\mathrm{F}}^{-1}-\phi^{-1} L_{\mathrm{O}}^{-1}, \\
& \xi>0, \tau=0^{+}: \quad H=\beta^{-1} E_{0} \theta(\xi, \tau)+L_{\mathrm{F}}^{-1}-1, \quad Z=L_{\mathrm{F}}^{-1}-\phi^{-1} L_{\mathrm{O}}^{-1} .
\end{aligned}
$$

These two equations (33) and (34), which are free from chemical sources, apply to the whole fluid field. They must be complemented by one of the equations (28) involving the reaction term $\Omega$.

\subsection{Near equidiffusivity approximation}

To ensure stability of the flame, we assume that both Lewis numbers are very close to unity, more precisely we define $l_{\mathrm{O}}=\beta\left(L_{\mathrm{O}}-1\right)$ and $l_{\mathrm{F}}=\beta\left(L_{\mathrm{F}}-1\right)$ to be of order unity. This is consistent with our previous scaling of the independent variables, since, as pointed out by Joulin [9], for a light limiting reactant, such that $0<1-L_{\mathrm{F}} \sim 1$, the evolution of the flame occurs in a very large timescale of order $\beta^{2}\left(1-\sqrt{L_{\mathrm{F}}}\right)^{2} t_{\mathrm{p}}$ and the dynamics of the kernel becomes quasi-steady, while in the opposite case, $0<L_{\mathrm{F}}-1 \sim 1$, the evolution time is so short that even the reaction zone is unsteady [3].

In the asymptotic limit $\beta \rightarrow \infty$ the reaction zone appears as a surface of discontinuity located at $\xi=\xi_{\mathrm{f}}(\tau)$ (in the following the subindex $f$ will refer to conditions at the flame, which will be time dependent), and the reaction term, $\Omega$, can be written in terms of the Dirac delta function as $m(\tau) \delta\left(\xi-\xi_{\mathrm{F}}\right)$, where $m(\tau)$ stands for the nondimensional mass flux of fuel reaching the flame per unit surface and time. We seek the solution as an expansion in powers of $\beta^{-1}$ in the form $\Theta=\Theta_{0}+\beta^{-1} \Theta_{1}+\cdots$, where $\Theta=(\varphi, X, Y, H, Z, m)$.

From the definition of $H$ and $Z$ we obtain the following relations

$$
\begin{aligned}
& H_{0}=\varphi_{0}+Y_{0}, \quad Z_{0}=Y_{0}-X_{0}, \\
& H_{1}=\varphi_{1}+Y_{1}-l_{\mathrm{F}} Y_{0}, \quad Z_{1}=Y_{1}-X_{1}-l_{\mathrm{F}} Y_{0}+l_{\mathrm{O}} X_{0} .
\end{aligned}
$$

The leading terms $H_{0}$ and $Z_{0}$ are given by the solution of (33)-(37) with $L_{\mathrm{F}}$ and $L_{\mathrm{O}}$ replaced by 1 . Then,

$$
H_{0}=\beta^{-1} E_{0} \theta(\xi, \tau), \quad Z_{0}=1-\phi^{-1} .
$$

The fuel concentration at leading order is given by $Y_{0}=0$ in the burnt side of the flame $\left(0<\xi<\xi_{\mathrm{f}}\right)$, while in the fresh gas side $\left(\xi>\xi_{\mathrm{f}}\right)$ is given by the solution of

$\frac{\partial Y_{0}}{\partial \tau}=\Delta Y_{0} \quad$ in $\xi_{\mathrm{f}}<\xi<\infty$,

$Y_{0}\left(\xi_{\mathrm{f}}, \tau\right)=0, \quad Y_{0}(\infty, \tau)=Y_{0}(\xi, 0)=1, \quad \frac{\partial Y_{0}\left(\xi_{\mathrm{f}}, \tau\right)}{\partial \xi}=m_{0}(\tau)$, 
which determines the distribution of the fuel mass fraction and the flame position in terms of $m_{0}(\tau)$. The last boundary condition comes from the jump relation across the thin reaction zone, or flame sheet. Once $Y_{0}$ and $\xi_{\mathrm{f}}$ have been computed we can obtain $\varphi_{0}$ and $X_{0}$ from (38) and (39). However, at this stage $m_{0}(\tau)$ is still an unknown function, which will be only determined from the analysis of the reaction zone.

Taking into account that $Z_{0}=1-\phi^{-1}$ we can write $H_{1}$ and $Z_{1}$ in the form

$$
\begin{aligned}
& H_{1}(\xi, \tau)=-l_{\mathrm{F}} V(\xi, \tau), \\
& Z_{1}(\xi, \tau)=-\left(l_{\mathrm{F}}-l_{\mathrm{O}}\right) V(\xi, \tau)+l_{\mathrm{O}}\left(\phi^{-1}-1\right),
\end{aligned}
$$

where $\mathrm{V}$ is the solution of the problem

$$
\begin{array}{lc}
\frac{\partial V}{\partial \tau}-\Delta V=\frac{\partial Y_{0}}{\partial \tau} & \text { in } 0<\xi<\infty \\
\frac{\partial V(0, \tau)}{\partial \xi}=0, & V(\infty, \tau)=V(\xi, 0)=1 .
\end{array}
$$

Behind the reaction zone we admit the existence of chemical equilibrium, i.e. $(X \cdot Y)_{\mathrm{f}}=0$. Since $\phi<1$ we obtain $Y_{0, \mathrm{f}}=0$ and $X_{0, \mathrm{f}}=\phi^{-1}-1$, which leads to $Y_{1, \mathrm{f}}=0$ and $X_{1, \mathrm{f}}=l_{0}\left(\phi^{-1}-1\right)-Z_{1, \mathrm{f}}$, or, from (42), $X_{1, \mathrm{f}}=\left(l_{\mathrm{F}}-l_{\mathrm{O}}\right) V_{\mathrm{f}}$.

\subsection{Reaction zone structure}

To analyse the reaction zone we introduce the stretched variable $\eta=\varepsilon^{-1}\left(\xi-\xi_{\mathrm{f}}\right)$ and expand $\varphi, X$ and $Y$ as

$$
\begin{aligned}
& \varphi=\beta^{-1} E_{0} \theta_{\mathrm{f}}+\varepsilon \psi+\cdots, \\
& X=\left(\phi^{-1}-1\right)+\varepsilon \tilde{X}+\cdots, \\
& Y=\varepsilon \tilde{Y}+\cdots,
\end{aligned}
$$

where the small parameter $\varepsilon$, to be defined below in (46), is a measure of the reaction zone thickness.

The inner and outer solutions must match as $\eta \rightarrow \pm \infty$ and $\xi \rightarrow \xi_{\mathrm{f}}^{ \pm}$, respectively, leading to

$\psi \rightarrow \beta^{-1} E_{0} \theta_{\xi, \mathrm{f}} \eta-(\varepsilon \beta)^{-1} l_{\mathrm{F}} V_{\mathrm{f}}, \quad \psi \rightarrow \beta^{-1} E_{0} \theta_{\xi, \mathrm{f}} \eta-(\varepsilon \beta)^{-1} l_{\mathrm{F}} V_{\mathrm{f}}-m_{0} \eta$,

$\tilde{X} \rightarrow(\varepsilon \beta)^{-1}\left(l_{\mathrm{F}}-l_{\mathrm{O}}\right) V_{\mathrm{f}}, \quad \tilde{X} \rightarrow(\varepsilon \beta)^{-1}\left(l_{\mathrm{F}}-l_{\mathrm{O}}\right) V_{\mathrm{f}}+m_{0} \eta$,

$\tilde{Y} \rightarrow 0, \quad \tilde{Y} \rightarrow m_{0} \eta$,

as $\eta \rightarrow-\infty$ and $\eta \rightarrow \infty$, respectively.

Introducing the expansions (44) in the expression for the reaction rate (32) it becomes apparent that it is convenient to define

$$
\varepsilon=\frac{1}{\beta}\left(1+\alpha \beta^{-1} E_{0} \theta_{\mathrm{f}}\right)^{2} .
$$

When equations (28) are written in terms of the inner variables we obtain $\tilde{X}=\tilde{Y}+$ $\left(1+\alpha \beta^{-1} E_{0} \theta_{\mathrm{f}}\right)^{-2}\left(l_{\mathrm{F}}-l_{0}\right) V_{\mathrm{f}}$ and two equations for $\psi$ and $\tilde{Y}$. For given values of $E_{0}, \beta$, $\alpha, n_{\mathrm{F}}$ and $l_{\mathrm{F}}$, these equations, with the boundary conditions (45), and problems (40) and (43), for $Y_{0}$ and $V$, respectively, constitute a closed, nonlinear, nonsteady, free-boundary problem, in which $m_{0}(\tau)$ is an eigenfunction, to be solved in order to obtain the time-position history of the front, $\xi_{\mathrm{f}}(\tau)$, which will determine whether the flame initiation is successful or not.

This problem can be greatly simplified since we can expect that the important events for flame initiation will not occur in the rapid stage taking place at the very beginning after the 
deposition of energy, when the flame is located at the core of the hot spot and $\theta_{\mathrm{f}}$ takes very high values, but during the longer period in which $\theta_{\mathrm{f}}$ has decreased to values of order unity. Thus, if we take into account that $E_{0} \sim 1$, and assume $\theta_{\mathrm{f}}$ to be small compared with $\beta$, we can write $1+\alpha \beta^{-1} E_{0} \theta_{\mathrm{f}} \approx 1$ in first approximation, which implies $\varepsilon=1 / \beta$. Moreover, in this slow stage we can assume $\xi_{\mathrm{f}} \gg \varepsilon$ and that the evolution time of the flame is not very short compared with $t_{\mathrm{p}}$, leading to the well-known reaction-diffusion balance for $\psi$ and $\tilde{Y}$ :

$$
-\frac{\mathrm{d}^{2} \psi}{\mathrm{d} \eta^{2}}=\frac{\mathrm{d}^{2} \tilde{Y}}{\mathrm{~d} \eta^{2}}=\frac{1}{2 \Gamma\left(n_{\mathrm{F}}+1\right)} \exp \left(E_{0} \theta_{\mathrm{f}}\right) \tilde{Y}^{n_{\mathrm{F}}} \mathrm{e}^{\psi} .
$$

From the first equation (47) and (45) we obtain $\psi=-\tilde{Y}+\beta^{-1} E_{0} \theta_{\xi, \mathrm{f}} \eta-l_{\mathrm{F}} V_{\mathrm{f}}$, which allows us to reduce the system (47) to a single equation for $\tilde{Y}$. Using $x=m_{0} \eta$ as new independent variable and defining

$$
\mu=-\frac{\beta^{-1} E_{0} \theta_{\xi, \mathrm{f}}}{m_{0}}>0 \quad \text { and } \quad D=\frac{1}{m_{0}^{2}} \exp \left(E_{0} \theta_{\mathrm{f}}-l_{\mathrm{F}} V_{\mathrm{f}}\right),
$$

the problem describing the reaction zone structure can be written as

$$
\begin{aligned}
& \frac{\mathrm{d}^{2} \tilde{Y}}{\mathrm{~d} x^{2}}=\frac{D}{2 \Gamma\left(n_{\mathrm{F}}+1\right)} \tilde{Y}^{n_{\mathrm{F}}} \mathrm{e}^{-\mu x-\tilde{Y}} ; \\
& \tilde{Y} \rightarrow 0 \quad \text { for } x \rightarrow-\infty, \quad \tilde{Y}-x \rightarrow 0 \quad \text { for } x \rightarrow+\infty,
\end{aligned}
$$

as in the premixed flame regime found by Liñán [13]. The Damköhler number, $D$, plays the role of an eigenvalue and should be determined as a function of the parameters $n_{\mathrm{F}}$ and $\mu$. However, notice that when $\beta^{-1} \theta_{\mathrm{f}} \ll 1$ the space derivative satisfies also $-\beta^{-1} \theta_{\xi, \mathrm{f}} \ll 1$, leading to $\mu \ll 1$. For these values the solution of (49) can be sought as an expansion in powers of $\mu$ obtaining $D=1+\mathcal{O}(\mu)$. So that we can take $D=1$ and from the second relation (48) it follows that

$$
m_{0}(\tau)=\exp \left(\frac{E_{0} \theta_{\mathrm{f}}-l_{\mathrm{F}} V_{\mathrm{f}}}{2}\right) .
$$

Therefore to obtain the evolution of the flame-front position, $\xi_{\mathrm{f}}(\tau)$, we need to solve (40) and (43) together with the above relation for $m_{0}(\tau)$. The complete mathematical problem, without subindexes, can be written as

$$
\begin{aligned}
& \frac{\partial Y}{\partial \tau}-\frac{1}{\xi^{j}} \frac{\partial}{\partial \xi}\left(\xi^{j} \frac{\partial y}{\partial \xi}\right)=0, \quad \text { in } \xi_{\mathrm{f}}<\xi<\infty, \\
& Y\left(\xi_{\mathrm{f}}, \tau\right)=0, \quad Y(\infty, \tau)=Y\left(\xi>\xi_{\mathrm{f}}, 0\right)=1, \\
& \frac{\partial V}{\partial \tau}-\frac{1}{\xi^{j}} \frac{\partial}{\partial \xi}\left(\xi^{j} \frac{\partial V}{\partial \xi}\right)=\frac{\partial Y}{\partial \tau}, \quad \text { in } 0<\xi<\infty, \\
& V_{\xi}(0, \tau)=0, \quad V(\infty, \tau)=V(\xi, 0)=1, \\
& Y_{\xi}\left(\xi_{\mathrm{f}}, \tau\right)=\exp \left(\frac{E_{0} \theta\left(\xi_{\mathrm{f}}, \tau\right)-l_{\mathrm{F}} V\left(\xi_{\mathrm{f}}, \tau\right)}{2}\right),
\end{aligned}
$$

with $\xi_{\mathrm{f}}(0)=0, Y\left(\xi<\xi_{\mathrm{f}}, \tau\right)=0$ and $\theta(\xi, \tau)=\mathrm{e}^{-\xi^{2} / 4 \tau} / \tau^{(1+j) / 2}$. Notice that equation (53) defines $\xi_{\mathrm{f}}$ implicitly since both $Y_{\xi}\left(\xi_{\mathrm{f}}, \tau\right)$ and $V\left(\xi_{\mathrm{f}}, \tau\right)$ depend on $\xi_{\mathrm{f}}$. This problem contains only two parameters: $E_{0}$, nondimensional measure of the external initiating energy, and $l_{\mathrm{F}}=\beta\left(L_{\mathrm{F}}-1\right)$. 


\subsection{Numerical solution and results}

To solve numerically the unsteady free-boundary problem (51)-(53) we use a marching in time algorithm that can be outlined as follows. We start by rewriting the problem (51) in terms of a new space variable $\eta=\xi-\xi_{\mathrm{f}}$. As result, the integration domain changes to $0<\eta<\infty$ with fixed boundaries and the velocity of the front, $\dot{\xi}_{\mathrm{f}}$, appears explicitly in the problem and it will be considered as an unknown instead of the position of the front, $\xi_{\mathrm{f}}$, that will be computed from $\int_{0}^{\tau} \dot{\xi}_{\mathrm{f}}\left(\tau^{\prime}\right) \mathrm{d} \tau^{\prime}$. Let us assume that the solution for $\tau_{1}<\tau_{2}<\cdots<\tau_{k-1}$ is known. For $\tau_{k}=\tau_{k-1}+\Delta \tau$ we choose an initial guess for $\dot{\xi}_{\mathrm{f}}\left(\tau_{k}\right)$, say $v_{k}^{0}$, and compute an approximation to $\xi_{\mathrm{f}}\left(\tau_{k}\right)$ as $s_{k}^{0}=\xi_{\mathrm{f}}\left(\tau_{k-1}\right)+\Delta \tau\left(v_{k}^{0}+\dot{\xi}_{\mathrm{f}}\left(\tau_{k-1}\right)\right) / 2$. The accurate value of $\xi_{\mathrm{f}}\left(\tau_{k}\right)$ is obtained according to the following iterative scheme.

For $v=1,2, \ldots$,

1. solve problem (51), with $\dot{\xi}_{\mathrm{f}}\left(\tau_{k}\right)$ and $\xi_{\mathrm{f}}\left(\tau_{k}\right)$ replaced by $v_{k}^{\nu-1}$ and $s_{k}^{\nu-1}$, to obtain $Y^{\nu-1}$ and $Y_{\xi}^{\nu-1}\left(s_{k}^{\nu-1}, \tau_{k}\right)$

2. solve problem (52) to obtain $V^{\nu-1}$;

3. solve equation (53) using the Newton method to obtain $v_{k}^{v}$, which requires to compute $\partial Y / \partial \dot{\xi}_{\mathrm{f}}$ and $\partial V / \partial \dot{\xi}_{\mathrm{f}}$, and compute a new approximation to $\xi_{\mathrm{f}}\left(\tau_{k}\right)$ as $s_{k}^{v}=\xi_{\mathrm{f}}\left(\tau_{k-1}\right)+$ $\Delta \tau\left(v_{k}^{v}+\dot{\xi}_{\mathrm{f}}\left(\tau_{k-1}\right)\right) / 2$.

To initiate this procedure we need the solution for $\tau \ll 1$ that can be obtained as a quasisimilar solution in the form $\xi_{\mathrm{f}}(\tau)=\sqrt{\tau} a(\tau), Y=Y(x, \tau)$ and $V=V(x, \tau)$ where $a(\tau)$ is an unknown function, determined from (53), and the similarity variable $x$ is defined as $x=\left(\xi-\xi_{\mathrm{f}}(\tau)\right) / \sqrt{\tau}$.

In figure 4 we have plotted the flame position-time histories, $\xi_{\mathrm{f}}(\tau)$, for several configurations. Figures $4(a)-(c)$ correspond to $l_{\mathrm{F}}=0$ and $j=0,1,2$, showing the effect of the geometry, while in figures $4(c)-(e)$ we illustrate the effect of the Lewis number $\left(l_{\mathrm{F}}=0,-2,2\right)$. In the spherical configuration there exists a well-defined critical value of the input energy, $E_{0, \mathrm{c}}$, such that for $E_{0}<E_{0, \mathrm{c}}$ the flame reaches a maximum radius and then starts to move back, collapsing to the origin at a quenching time $\tau_{\mathrm{q}}$. For supercritical values the flame radius grows continuously and, eventually, the flame propagates at a constant speed. The separatrix between both regimes, plotted with dashed line, corresponds to the critical value $E_{0, c}$, for which the problem attains the steady state and, hence, $\xi_{\mathrm{f}}$ tends to the Zel'dovich flame-ball radius given by $\xi_{\mathrm{f}, \mathrm{c}}=\mathrm{e}^{l_{\mathrm{F}} / 2}$. In the cylindrical and planar cases the problem does not admit a such steady state and therefore we cannot define a similar separatrix. However, we can observe two welldefined behaviours: quenching and self-propagation. For practical purposes it is convenient to define a critical value that can be used as a reference value to establish a division between both regimes. To this end we have defined $E_{0, \mathrm{c}}$ as the value that leads to quenching and such that $E_{0, \mathrm{c}}+10^{-4}$ leads to propagation. We have also plotted the corresponding trajectories with dashed line.

The flame speed $\dot{\xi}_{\mathrm{f}}$ is plotted in figure 5 for the same values and configurations as in figure 4. Notice that $\dot{\xi}_{\mathrm{f}}\left(\tau_{q}\right)=-\infty$ in the cylindrical and spherical cases, while $\dot{\xi}_{\mathrm{f}}\left(\tau_{q}\right)$ is finite in the planar case. This geometrical effect can also be observed in figure 4 .

Finally, figure 6 shows the critical value, $E_{0, c}$, dividing the quenching and propagation regimes as a function of the reduced Lewis number $l_{\mathrm{F}}$.

\section{Results and conclusions}

We have analysed, in the limit of large activation energy, two related problems: (i) the ignition of a gaseous reactive mixture resulting from the thermal nonuniformity produced by the deposition 
(a)

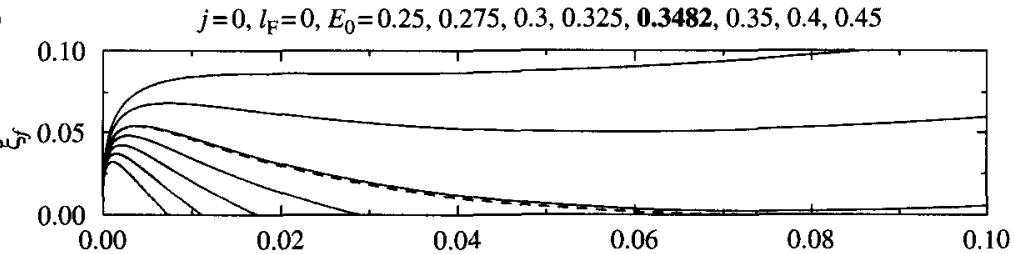

(b) $\quad j=1, l_{\mathrm{F}}=0, E_{0}=0.3,0.35,0.40 .45,0.5,0.5042,0.51,0.55$

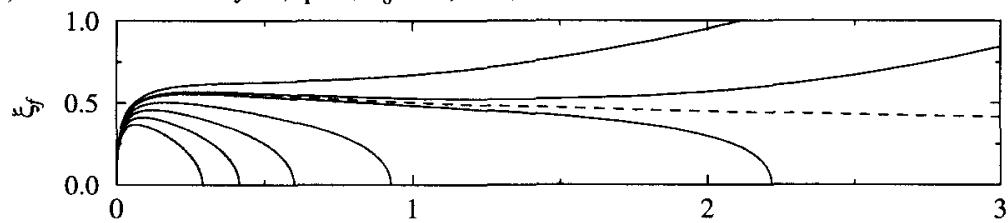

(c)

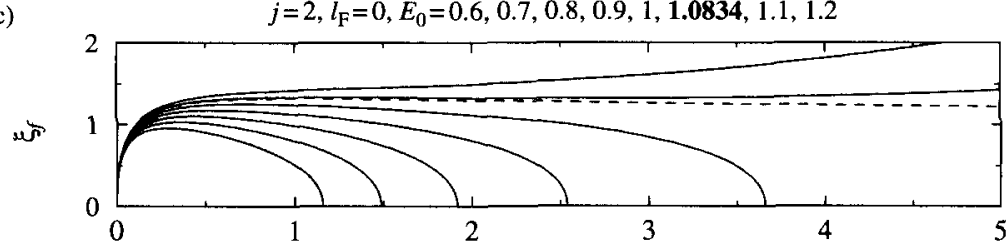

(d)

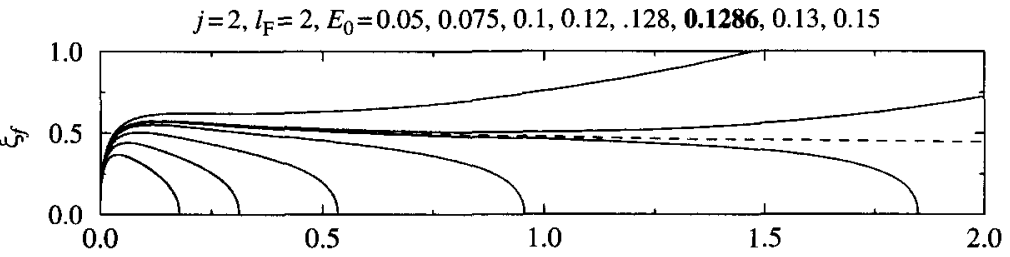

(e)

$j=2, l_{\mathrm{F}}=2, E_{0}=5,6,6.5,7,7.3,7.4088,7.5,8$

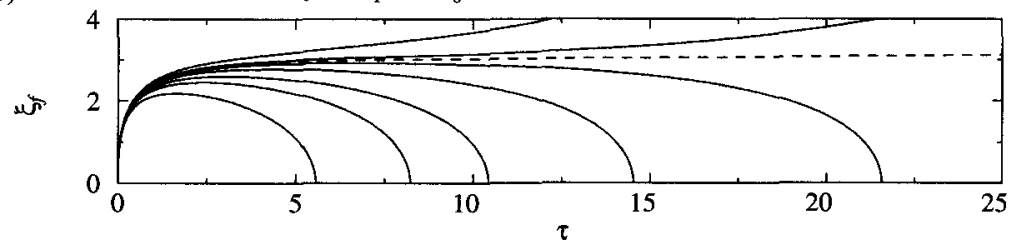

Figure 4. The position of the flame front, $\xi_{\mathrm{f}}$, as a function of time for several values of $E_{0}$, in five different configurations. The critical value is typeset in bold characters.

of energy in a small region of the gas, and (ii) the flame initiation problem subsequent to the ignition. We assume that the time required for the deposition of energy is large compared with the time of acoustic waves propagation but short compared with the characteristic time of heat conduction. Therefore, heat conduction becomes the dominant mechanism for energy transport and has to compete with the heat release by the chemical reaction, which has been triggered by the external addition of energy. The asymptotic analysis lead to a model to describe the ignition process containing a single parameter: the Damköhler number, $\delta$. The numerical results show that ignition only occurs for values of $\delta$ greater than a critical value, which is computed for the planar, cylindrical and spherical configurations.

For energy source with a size small compared with $l_{\mathrm{p}}$, the ignition, or local thermal runaway, is not a sufficient condition to ensure a successful flame initiation and propagation since a larger timescale is involved, in which we must account for the diffusion and consumption 

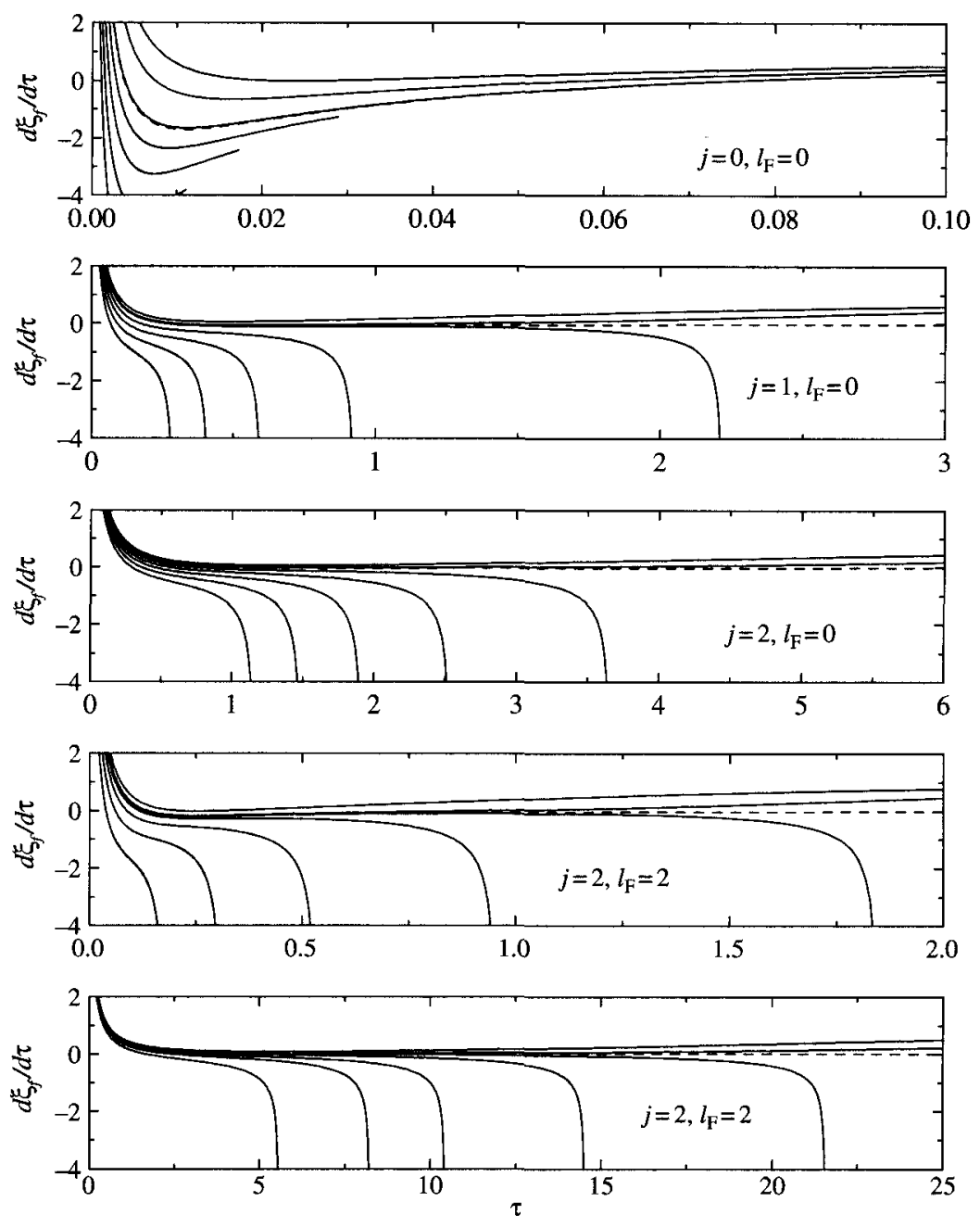

Figure 5. The velocity of the flame front, $\dot{\xi}_{f}$, as a function of time for the same values of $E_{0}$ and configurations as in figure 4.

of reactants, neglected during the ignition transient. To obtain, for energy sources of small size, conditions leading to flame propagation after ignition, we have omitted the description of the very early stage in which the ignition reaction zone evolves to a propagating flame front. Instead we have considered that a local source releases instantaneously a finite amount of energy giving rise to a well established flame front, whose dynamics will determine if the flame initiation is successful or not. This simplification is justified since the flame propagation occurs in a timescale much larger than the scale associated with the development of the flame kernel. We find that, for given physicochemical parameters of the mixture, the energy input must be greater than a critical value to attain the self-propagating regime. The results also reveals several features of the flame quenching: (i) it does not take place necessarily during the early stages of flame propagation; (ii) the quenching always occurs as a collapse of the flame at the position of the source; and (iii) the beginning of the receding movement of the flame is not associated with a very low frozen temperature. 
The critical value of the input energy obtained in section 3 must be seen as the minimum energy content of a hot spot required to produce a successful flame initiation. A hot spot of small size, measured with $r_{\mathrm{h}} / l_{\mathrm{p}}$, will require less energy to ignite the mixture, while for large hot spots the amount of energy for ignition will be also sufficient to ensure flame propagation. A simplified representation of this situation is given in figure 7 where we have plotted the minimum energy for flame initiation, $E_{0, \min }=\tilde{E}_{0, \min } / \beta^{-1} \rho c_{p}\left(T_{\mathrm{b}}-T_{0}\right)\left(4 \pi l_{\mathrm{p}}^{2}\right)^{(1+j) / 2}$, as a function of the hot-spot size, $r_{\mathrm{h}} / l_{\mathrm{p}}$, for $\beta=15, l_{\mathrm{F}}=0, n_{\mathrm{F}}=1, n_{\mathrm{O}}=0$ and $\alpha=0.8$. The straight lines correspond to $E_{0, \min }=E_{0, \mathrm{c}}$. The curves of minimum energy for ignition have been obtained assuming that the initial distribution of temperature in the hot spot is given by $T_{\mathrm{h}}(r)=T_{0}+\left(T_{\mathrm{m}}-T_{0}\right) \mathrm{e}^{-k r^{2}}$, with $k$ chosen to satisfy $r_{\mathrm{h}}^{2}=$ $-2 T_{\mathrm{m}} /\left(\mathrm{d}^{2} T_{\mathrm{h}} / \mathrm{d} r^{2}\right)_{r=0}$. The dimensionless energy content of the hot spot is then given by $E_{0}=\beta\left(\left(1-T_{0} / T_{\mathrm{m}}\right) / 4\right)^{(1+j) / 2}\left(r_{\mathrm{h}} / l_{\mathrm{p}}\right)^{1+j}\left(T_{\mathrm{m}}-T_{0}\right) /\left(T_{\mathrm{b}}-T_{0}\right)$, which, together with (17), determines the minimum energy for ignition, $E_{0, \mathrm{ig}}$, as a function of $r_{\mathrm{h}} / l_{\mathrm{p}}$. Obviously $E_{0, \min }=\min \left(E_{0, \mathrm{c}}, E_{0, \mathrm{ig}}\right)$. Thus for hot spots larger than the flame thickness the condition

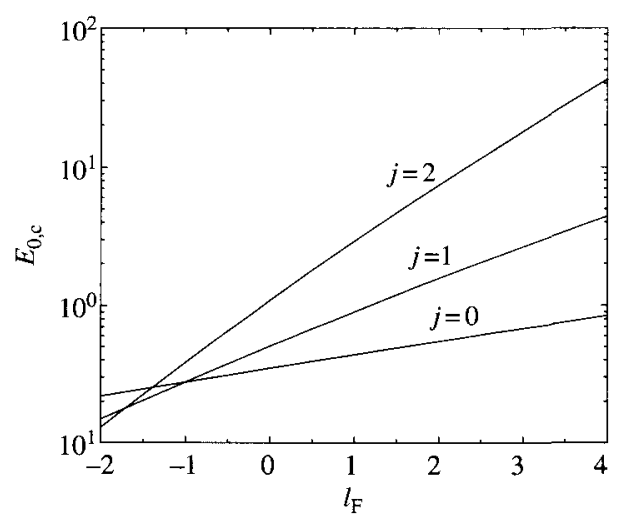

Figure 6. The critical energy $E_{0, c}$ as a function of the reduced Lewis number $l_{\mathrm{F}}$ for a nonstoichiometric mixture.

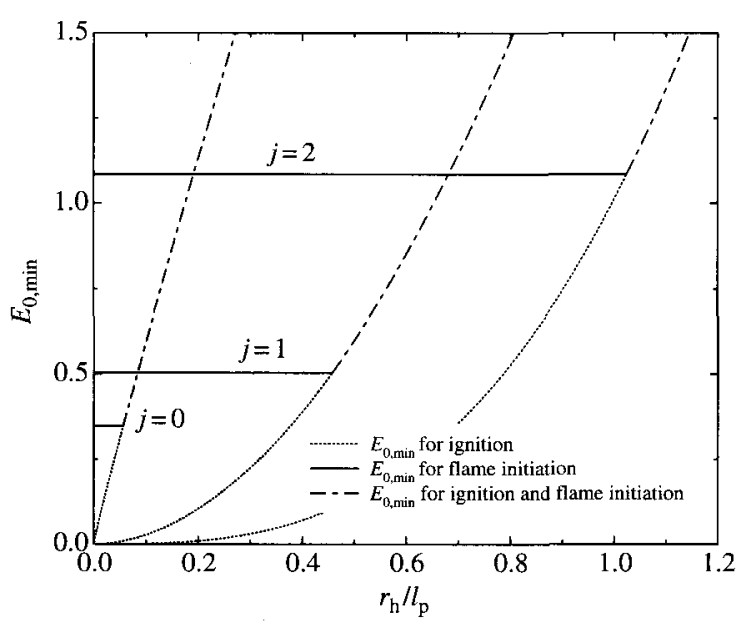

Figure 7. The minimum energy for flame initiation as a function of the hot-spot size, $r_{\mathrm{h}} / l_{\mathrm{p}}$, in the cylindrical and spherical configurations for $\beta=15, l_{\mathrm{F}}=0, n_{\mathrm{F}}=1, n_{\mathrm{O}}=0$ and $\alpha=0.8$. 
for ignition is also a sufficient condition for flame initiation, while for $r_{\mathrm{h}} \lesssim l_{\mathrm{p}}$ an additional increase in the ignition energy is required.

It should be pointed out that our analysis does not rest on the existence of the Zel'dovich flame ball and its unstable character, which obviously is included, and, hence, the analysis is not restricted to the spherical case but it also applies to the planar and cylindrical cases. On the other hand, we have determined not only the critical conditions leading to flame initiation but also we have given a description of the dynamics of the process in terms of the position-time histories of the flame.

Notice that the symmetrical calculations carried out here can not show the thermaldiffusive instabilities described by Sivashinsky [15] for $l_{\mathbf{F}}<-2$ although if carried out for $l_{\mathrm{F}}>21 / 2$ should show pulsating instabilities. The above theory can be extended to account for arbitrary Lewis numbers, noninstantaneous energy source and effects of variable density and flow-field. This will be considered elsewhere.

\section{Appendix. Minimum energy for flame initiation in a quasi-stoichiometric mixture}

When the initial mixture is quasi-stoichiometric, i.e. $\beta(1-\phi) \sim 1$, the analysis of section 3 can be extended with only minor changes. We start by writing the equivalence ratio as $\phi=1+\beta^{-1} \phi_{1}+\cdots$. The two first terms of the expansion for $H$ and $Z$ are now given by

$$
\begin{aligned}
& H_{0}=\varphi_{0}+Y_{0}, \quad H_{1}=-l_{\mathrm{F}} V(\xi, \tau), \\
& Z_{0}=0, \quad Z_{1}=\left(l_{\mathrm{O}}-l_{\mathrm{F}}\right) V(\xi, \tau)+\phi_{1},
\end{aligned}
$$

with $V(\xi, \tau)$ given by the solution of (43). Now both species vanish at the flame in first approximation and the condition $\left(X_{1} \cdot Y_{1}\right)_{\mathrm{f}}=0$ must hold. It is easy to check that $X_{1, \mathrm{f}}$ and $Y_{1, \mathrm{f}}$ are given in terms of $Z_{1, \mathrm{f}}$ by the following expressions

$$
X_{1, \mathrm{f}}=\frac{1}{2}\left(\left|Z_{1, \mathrm{f}}\right|-Z_{1, \mathrm{f}}\right), \quad Y_{1, \mathrm{f}}=\frac{1}{2}\left(\left|Z_{1, \mathrm{f}}\right|+Z_{1, \mathrm{f}}\right) .
$$

Notice that, as pointed out by Joulin [10], nonstoichiometric mixtures keep their character at the flame, but if the mixture is quasi-stoichiometric the preferential diffusion effects result in a mixture that close to the flame can be lean $\left(Z_{1, \mathrm{f}}<0\right)$ or rich $\left(Z_{1, \mathrm{f}}>0\right)$. For given values of $l_{\mathrm{F}}$ and $l_{\mathrm{O}}$ there exists a critical value of $\phi_{1}$, say $\phi_{\mathrm{c}}$, such that if $\phi_{1}=\phi_{\mathrm{c}}$ the mixture is locally stoichiometric $\left(Z_{1, \mathrm{f}}=0\right)$, i.e. the departure from the stoichiometric condition of the fresh mixture far from the flame is balanced by the preferential diffusion effects. Since the critical value, given by $\phi_{\mathrm{c}}(\tau)=\left(l_{\mathrm{F}}-l_{\mathrm{O}}\right) V_{\mathrm{f}}$, is time dependent the local stoichiometric character of the flame can change during the flame initiation process.

The analysis of the reaction zone structure is carried out by means of the stretched variable $\eta=\varepsilon^{-1}\left(\xi-\xi_{\mathrm{f}}\right)$ and the expansions $\varphi=\beta^{-1} E_{0} \theta_{\mathrm{f}}(\tau)+\varepsilon \psi+\cdots, Y=\varepsilon \tilde{Y}+\cdots$ and $X=\varepsilon \tilde{X}+\cdots$ After similar arguments to those of section 3.3 we can take $\varepsilon=1 / \beta$, and we find that the functions $\psi, \tilde{Y}$ and $\tilde{X}$ satisfy

$$
-\frac{\mathrm{d}^{2} \psi}{\mathrm{d} \eta^{2}}=\frac{\mathrm{d}^{2} \tilde{Y}}{\mathrm{~d} \eta^{2}}=\frac{\mathrm{d}^{2} \tilde{X}}{\mathrm{~d} \eta^{2}}=\omega_{0},
$$

and the following matching conditions:

$$
\begin{aligned}
& \psi \rightarrow \beta^{-1} E_{0} \theta_{\xi, \mathrm{f}} \eta+H_{1, \mathrm{f}}, \quad \psi \rightarrow \beta^{-1} E_{0} \theta_{\xi, \mathrm{f}} \eta+H_{1, \mathrm{f}}-m_{0} \eta, \\
& \tilde{X} \rightarrow X_{1, \mathrm{f}}, \quad \tilde{X} \rightarrow X_{1, \mathrm{f}}+m_{0} \eta, \\
& \tilde{Y} \rightarrow Y_{1, \mathrm{f}}, \quad \tilde{Y} \rightarrow Y_{1, \mathrm{f}}+m_{0} \eta,
\end{aligned}
$$

for $\eta \rightarrow-\infty$ and $\eta \rightarrow \infty$, respectively. The leading term of the reaction rate, $\omega_{0}$, is written as

$$
\omega_{0}=\frac{1}{2 G\left(\infty ; n_{\mathrm{F}}, n_{\mathrm{O}}, \phi_{1} / 2\right)} \exp \left(E_{0} \theta_{\mathrm{f}}\right) \tilde{Y}^{n_{\mathrm{F}}} \tilde{X}^{n_{\mathrm{O}}} \mathrm{e}^{\psi},
$$


with the function $G$ given by

$$
G\left(\eta ; n_{\mathrm{F}}, n_{\mathrm{O}}, a\right)=\int_{0}^{\eta}(|a|+a+t)^{n_{\mathrm{F}}}(|a|-a+t)^{n_{\mathrm{O}}} \mathrm{e}^{-t} \mathrm{~d} t,
$$

as result of the definition of $U_{\mathrm{p}}$ in the stoichiometric case. We readily obtain $\psi+\tilde{Y}=$ $\beta^{-1} E_{0} \theta_{\xi, \mathrm{f}} \eta+H_{1, \mathrm{f}}+Y_{1, \mathrm{f}}$ and $\tilde{Y}-\tilde{X}=Z_{1, \mathrm{f}}$, which allow us to write a single equation for $\tilde{Y}$. It is convenient to reduce it to a canonical form by using $x=m_{0} \eta$ and $S=\tilde{Y}-Y_{1, \mathrm{f}}$ as new independent and dependent variables, respectively, and defining

$$
\begin{aligned}
& \zeta=\frac{Z_{1, \mathrm{f}}}{2}, \quad g=\frac{G\left(\infty ; n_{\mathrm{F}}, n_{\mathrm{O}}, \zeta\right)}{G\left(\infty ; n_{\mathrm{F}}, n_{\mathrm{O}}, \phi_{1} / 2\right)}, \\
& \mu=\frac{-\beta^{-1} E_{0} \theta_{\xi, \mathrm{f}}}{m_{0}}, \quad D=\frac{g}{m_{0}^{2}} \exp \left(E_{0} \theta_{\mathrm{f}}+H_{1, \mathrm{f}}\right) .
\end{aligned}
$$

Then the problem for $S$ is found to be

$$
\begin{aligned}
& \frac{\mathrm{d}^{2} S}{\mathrm{~d} x^{2}}=\frac{D}{2 G\left(\infty ; n_{\mathrm{F}}, n_{\mathrm{O}}, \zeta\right)}(|\zeta|+\zeta+S)^{n_{\mathrm{F}}}(|\zeta|-\zeta+S)^{n_{\mathrm{O}}} \mathrm{e}^{-\mu x-S}, \\
& S \rightarrow 0 \quad \text { for } x \rightarrow-\infty, \quad S-x \rightarrow 0 \quad \text { for } x \rightarrow+\infty .
\end{aligned}
$$

This problem, as (49), only admits solution for an appropriate value of the Damköhler number, $D$, that must be computed for given values of $\mu, n_{\mathrm{F}}, n_{\mathrm{O}}$ and $\zeta$. For $\mu \ll 1$ it can be shown that $D=1+\mathcal{O}(\mu)$ and it follows that

$$
m_{0}(\tau)=g^{1 / 2} \exp \left(\frac{E_{0} \theta_{\mathrm{f}}+H_{1, \mathrm{f}}}{2}\right) .
$$

The problem to be solved is (51)-(53) with the above definition for $m_{0}(\tau)$ and the expressions of $H_{1, \mathrm{f}}$ and $Z_{1, \mathrm{f}}$ in terms of $V_{\mathrm{f}}$, i.e. $H_{1, \mathrm{f}}=-l_{\mathrm{F}} V_{\mathrm{f}}$ and $Z_{1, \mathrm{f}}=\left(l_{\mathrm{O}}-l_{\mathrm{F}}\right) V_{\mathrm{f}}+\phi_{1}$. The parameters involved in this case are $E_{0}, l_{\mathrm{F}}, l_{\mathrm{O}}, n_{\mathrm{F}}, n_{\mathrm{O}}$ and $\phi_{1}$, making difficult to give the result in the whole parameter space. As an example, we have considered the spherical configuration and the particular set of values $n_{\mathrm{f}}=n_{\mathrm{O}}=1, l_{\mathrm{O}}=0$ and $\phi_{1}=-2,-1,0,1,2$. In figure 8 we have plotted the value of the critical energy as a function of $l_{\mathrm{F}}$. The curves of $\xi_{\mathrm{f}}$ as function of $\tau$ for different values of $E_{0}$ and $l_{\mathrm{F}}$ are quite similar to those of figure 4 .

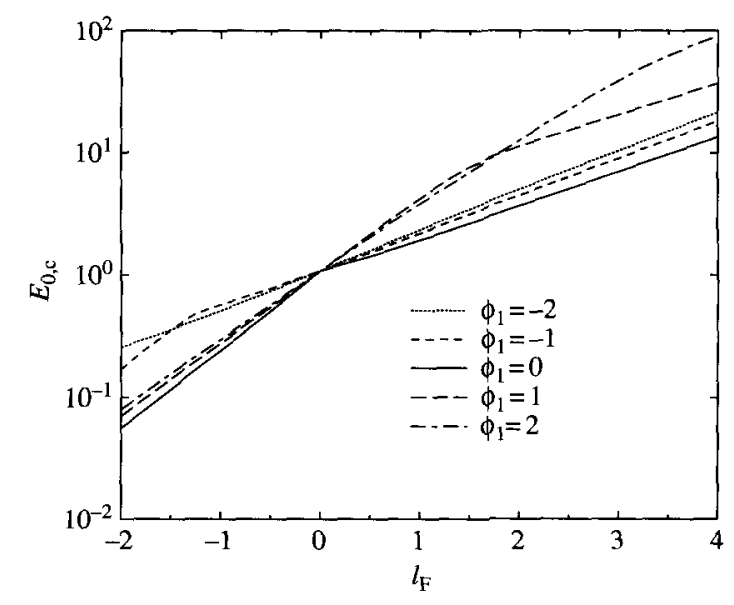

Figure 8. The critical energy $\left(\beta E_{0}\right)_{c}$ in the spherical configuration as a function of the reduced Lewis number $l_{\mathrm{F}}$ for a quasi-stoichiometric mixture with $l_{\mathrm{O}}=0, n_{\mathrm{F}}=n_{\mathrm{O}}=1$ and different values of $\phi_{1}$. 


\section{Acknowledgment}

This work has been partially supported by the Spanish Dirección General de Investigación of the Ministerio de Ciencia y Tecnología under contract number BFM2001-3691.

Berman V S, Riazantsev I S and Shevtsova V M 1981 Asymptotic analysis of the process of igniting a combustible gas mixture by thermal inhomogeneity J. Appl. Math. Mech. 44 60-4

Cambray P and Joulin G 1989 Stoichiometry effects in the point-source initiation of lean flames of a light fuel Combust. Sci. Technol. 65 167-79

Champion M, Deshaies B, Joulin G and Kinoshita K 1986 Spherical flame initiation: theory versus experiments for lean propano-air mixtures Combust. Flame 65 319-37

Deshaies B and Joulin G 1984 On the initiation of a spherical flame kernel Combust. Sci. Technol. 37 99-116

Dold J W 1985 Analysis of the early stage of thermal runaway Quart. J. Mech. Appl. Math. 38 361-87

Eckett C A, Quirk J J and Shepherd J E 2000 The role of unsteadiness in direct initiation of gaseous detonations J. Fluid Mech. 421 147-83

He L 2000 Critical conditions for spherical flame initiation in mixtures with high Lewis numbers Combust. Theory Modelling 4 159-72

He $\mathrm{L}$ and Clavin P 1994 On the direct initiation of gaseous detonations by an energy source J. Fluid Mech. 277 $227-48$

Joulin G 1985 Point source initiation of lean spherical flames of light reactants: an asymptotic theory Combust. Sci. Technol. 43 99-113

Joulin G 1987 Preferential diffusion and the initiation of lean flames of light fuels SIAM J. Appl. Math. 47 998-1016

Kapila A K 1981 Evolution of deflagration in a cold combustible subjected to a uniform energy flux Int. J. Engng Sci. $19495-509$

Kassoy D R, Kapila A K and Stewart D S 1989 A unified formulation for diffusive and nondiffusive thermal explosion theory Combust. Sci. Technol. $6333-43$

Liñán A 1974 The asymptotic structure of counterflow diffusion flames for large activation energies Acto Astronaut. 1 1007-39

Short M 1997 On the critical conditions for the initiation of a detonation in a nonuniformly perturbed reactive fluid SIAM J. Appl. Math. 57 1242-80

Sivashinsky G I 1977 Diffusional-thermal theory of cellular flames Combust. Sci. Technol. 15 137-46

Vázquez-Espí C and Liñán A 2001 Fast, nondiffusive ignition in a gaseous reacting mixture subject to a poin energy source Combust. Theory Modelling 5 485-98

Zel'dovich Y B, Barenblatt G I, Librovich B V and Makhviladze G M 1985 Mathematical Theory of Combustion and Explosions (New York: Plenum) 